\title{
Induction of Fanconi Anemia Cellular Phenotype in Human 293 Cells by Overexpression of a Mutant FAC Allele
}

\author{
Hagop Youssoufian, ${ }^{\star}$ Youlin Li, ${ }^{*}$ Mary E. Martin, ${ }^{\star}$ and Manuel Buchwald ${ }^{\ddagger}$ \\ *Department of Medicine, Hematology-Oncology Division, Brigham and Women's Hospital, and Harvard Medical School, Boston, \\ Massachusetts 02115; and $\doteqdot$ Department of Genetics, Research Institute, The Hospital for Sick Children, and Department of Molecular and \\ Medical Genetics, University of Toronto, Toronto, Ontario, Canada M5G 1 X8
}

\begin{abstract}
The polypeptide encoded by the Fanconi anemia (FA) complementation group $\mathrm{C}$ gene, $F A C$, binds to a group of cytoplasmic proteins in vitro and may form a multimeric complex. A known mutant allele of FAC resulting from the substitution of Pro for Leu at codon 554 fails to correct the sensitivity of FA group $\mathrm{C}$ cells to mitomycin $\mathrm{C}$. We reasoned that overexpression of the mutant protein in a wildtype cellular background might induce the FA phenotype by competing with endogenous FAC for binding to the accessory proteins. After stable transfection of 293 cells with wild-type and a mutant FAC allele containing the L554P substitution, four independent clones that expressed fourto-fifteen fold higher levels of transcript from the mutant transgene relative to the endogenous FAC gene showed hypersensitivity to mitomycin $\mathrm{C}$. By contrast, both parental and $\mathrm{FAC}$-overexpressing cells maintained their relative resistance to mitomycin C. No differences in the biosynthesis, subcellular localization and protein interactions of the normal and mutant proteins were detected. The induction of the FA phenotype in this system is compatible with the competition hypothesis and provides support for a functional role of the FAC-binding proteins in vivo. (J. Clin. Invest. 1996. 97:957-962.) Key words: multimeric complex • GST fusion • transfection • phenotype $\bullet$ mitomycin C
\end{abstract}

\section{Introduction}

Fanconi anemia $(\mathrm{FA})^{1}$ is a rare autosomal recessive disorder characterized by congenital malformations, bone marrow failure, and the development of malignant disorders. The cellular hallmark of FA is spontaneous chromosomal fragility and its potentiation with bifunctional alkylating agents, such as mitomycin C (MMC) and diepoxybutane (1). The dual features of genomic instability and cancer predisposition have suggested that the fundamental defect in FA is in the repair of DNA le-

Address correspondence to Hagop Youssoufian, M.D., Brigham and Women's Hospital, 221 Longwood Avenue, LMRC 620, Boston, MA 02115. Phone: 617-732-5464; FAX: 617-739-3324.

Received for publication 27 September 1995 and accepted in revised form 21 November 1995.

1. Abbreviations used in this paper: FA, Fanconi anemia; FA-C, Fanconi anemia complementation group C; FABP, FAC binding protein; FAC, protein defective in FA-C; GST, glutathione $S$-transferase; MMC, mitomycin C.

J. Clin. Invest.

(C) The American Society for Clinical Investigation, Inc.

0021-9738/96/02/0957/06 \$2.00

Volume 97, Number 4, February 1996, 957-962 sions (2). However, in view of the genetic heterogeneity of FA $(3,4)$, it is possible that alterations in a number of independent biochemical pathways could give rise to the FA phenotype. Indeed, the recent localization of the FAC polypeptide, deficient in complementation group C (FA-C) cells, to the cytoplasm of mammalian cells has cast doubt on the hypothesis of defective DNA repair in FA-C $(5,6)$.

The FAC gene was isolated by virtue of its ability to rescue FA-C from the cytotoxicity of bifunctional crosslinking agents (7). The full-length cDNA encodes a polypeptide of 558 amino acids with a predicted molecular mass of $\sim 63 \mathrm{kD}$, which does not show significant homologies to other proteins. The gene is expressed ubiquitously in most mammalian cells, albeit at low levels. Additional genetic evidence for FAC in the pathobiology of FA was provided by the identification of intragenic mutations (7-11), including a substitution of proline for leucine at codon 554 (L554P) that completely abolished the ability of the protein to correct the hypersensitivity of a FA-C cell line to MMC (12).

Recently, we demonstrated that a group of cytoplasmic proteins (called FAC-binding proteins, or FABPs) are capable of binding specifically to a recombinant form of FAC immobilized to protein A-agarose beads (13). Yamashita et al. (5) have also observed a similar association of FAC with cellular proteins in immunocomplexes. Although these studies suggest that FAC is capable of forming a multimeric complex in vitro, the biological significance of these associations is unclear. In the course of studying the mechanism of inactivation of FAC by the L554P mutation, we reasoned that overexpression of the mutant protein in a non-FA cellular background might induce the FA phenotype by competing with endogenous FAC for binding to the FABPs. Here we demonstrate the induction of hypersensitivity to MMC in cells that were previously resistant to this agent by overexpression of an FAC transgene containing the L554P mutation.

\section{Methods}

Cell culture and transfection. COS-1 and adenovirus-transformed human kidney 293 cells (14) were grown in Dulbecco's Minimal Essential Medium (DME) and 10\% heat-inactivated fetal bovine serum (FBS). Epstein Barr virus-transformed lymphoblasts were grown in RPMI-15\% FBS. For transient transfections, COS-1 cells grown to $\sim 50 \%$ confluence were transfected with supercoiled plasmid DNA in a final concentration of $1 \mu \mathrm{g} / \mathrm{ml}$ using DEAE-dextran. For stable transfections, confluent 293 cells were split 1:5 into 100-mm dishes $24 \mathrm{~h}$ before transfection with a calcium phosphate precipitate containing $20 \mu \mathrm{g}$ of recombinant pED6 (15) and $2 \mu \mathrm{g}$ of pPGKneo (for neomycin selection). Cells were exposed to $0.4 \mu \mathrm{g} / \mathrm{ml} \mathrm{G} 418$ (GIBCO Laboratories, Grand Island, NY) during selection. Approximately 14 individual G418-resistant colonies were isolated with cloning rings after $3 \mathrm{wk}$ and grown to confluence in the same concentration of G418.

Immunofluorescence microscopy. $16 \mathrm{~h}$ after transfection cells 
were replated on sterile glass coverslips. $48 \mathrm{~h}$ after transfection cells were washed twice in PBS, $1 \%$ bovine serum albumin, fixed in $3.5 \%$ paraformaldehyde, and incubated sequentially with a 1:5,000 dilution of polyclonal antiserum raised against a recombinant fusion protein encoding glutathione $S$-transferase (GST) at the amino terminus and FAC (amino acids 6 to 558) at the carboxy-terminus, as described previously (6), followed by incubation with fluorescein-conjugated goat anti-rabbit IgC (Cappel, West Chester, PA).

Pulse-chase and immunoprecipitation. For pulse-chase studies, transfected COS-1 cells grown in 60-mm dishes were metabolically labeled with Expre ${ }^{35} \mathrm{~S}^{35} \mathrm{~S}$ label $(0.2 \mathrm{mCi} / \mathrm{ml}$; Dupont, Wilmington, DE) for $30 \mathrm{~min}$ in cysteine- and methionine-deficient DMEM. The labeled cells were washed rapidly with DME, and the radiolabel was chased by incubation in DME-10\% FBS. Cells were washed with ice-cold PBS and lysed in buffer A ( $20 \mathrm{mM}$ tris [pH 8.0], $50 \mathrm{mM} \mathrm{NaCl}, 0.5 \%$ deoxycholate, $1 \% \mathrm{NtP}-40$ ) and a cocktail of protease inhibitors. After centrifugation of the lysates for $5 \mathrm{~min}$ at $1,000 \mathrm{~g}$, nuclear and cytosolic extracts were immunoprecipitated sequentially with anti-FAC antibody for $16 \mathrm{~h}$ at $4^{\circ} \mathrm{C}$ and protein A-agarose beads for $2 \mathrm{~h}$ (BioRad, Richmond, CA). The unbound supernatant was subjected to a second round of immunoprecipitations to maximize the recovery of the antigen. The combined immunocomplexes were washed three times with NET-gel buffer (16), boiled in 1x Laemmli buffer in the presence of $200 \mathrm{mM}$ dithiothreitol, and analyzed by electrophoresis on denaturing 10\% SDS-polyacrylamide gels and autoradiography.

Expression of GST fusion proteins and FABP-binding assay. The entire coding sequence of FAC beginning with the second amino acid was inserted into the BamHI and EcoRI sites of pGEX2TK (Pharmacia, Piscataway, NJ) by a two-step process. (Details of these constructions will be provided upon request.) A 280-bp PstI-XbaI fragment from the carboxy-terminal coding region of FAC-L554P was substituted for the corresponding fragment in pGEX2TK-FAC. Procedures for bacterial expression, solubilization of inclusion bodies, and glutathione-agarose affinity purification was performed essentially as described (17).

For protein interaction studies, 293 cells were metabolically labeled with Expre ${ }^{35} \mathrm{~S}^{35} \mathrm{~S}$ label $(0.2 \mathrm{mCi} / \mathrm{ml})$ for $3 \mathrm{~h}$ in cysteine- and methionine-deficient DME. After washing in cold PBS, $\sim 5 \times 10^{6}$ were lysed in buffer A containing protease inhibitors and fractionated into nuclear and cytosolic extracts. Extracts were incubated for $1 \mathrm{~h}$ at $4^{\circ} \mathrm{C}$ with $\sim 20 \mu$ l glutathione-agarose beads in a final detergent concen- tration of $0.125 \%$ deoxycholate and $0.25 \%$ NP- 40 . After washing three times in the incubation buffer, samples were boiled and analyzed by SDS-PAGE.

RT-PCR of gene-specific products. Reverse transcription was performed using $6 \mathrm{mg}$ of DNaseI-treated cytoplasmic RNA, avian myeloblastosis virus reverse transcriptase, and oligo-dT primer (Collaborative Research, Lexington, MA). The product of the first-strand synthesis was divided into two parts and amplified by PCR using Taq polymerase and $50 \mathrm{pmol}$ of each FAC-specific primer. The primers were: common forward primer (5'-GGACCTGCAGACGGTAGC$\left.3^{\prime}\right), 3^{\prime}$ reverse primer corresponding to the wild-type FAC sequence (5'-CTAGACTTGAGTTCGCAGCT-3' [3' PCR primer "W"]), and $3^{\prime}$ reverse primer corresponding to the mutant sequence (5'-CTAGACTTGAGTTCGCGGC-3' [mutation underlined; 3' PCR primer "M"]). An annealing temperature of $58^{\circ} \mathrm{C}$ was found to be the most discriminatory. Amplifications were done for 30 cycles, precipitated with ethanol, and electrophoresed on a $1.2 \%$ agarose gel.

Determination of cell survival. Cell survival in response to crosslinking agents were performed by incubation of $1 \times 10^{4}$ cells with a range of concentrations of MMC (Sigma Chemical Co., St. Louis, $\mathrm{MO}$ ) for $5 \mathrm{~d}$ and counting of viable cells by trypan blue exclusion.

\section{Results}

No alterations in subcellular localization. We have previously demonstrated that the FAC polypeptide is cytoplasmic in a panel of mammalian cells, and placement of a nuclear localization signal from the SV-40 large T-antigen directs the chimeric protein to the nuclei of transiently transfected COS cells. (6) To evaluate the effect of the L554P mutation on the subcellular location of the protein, we subcloned either wild-type or mutant FAC cDNA in the mammalian expression vector pED6 (15) for transfection into COS- 1 cells. After $48 \mathrm{~h}$, transfected cells were analyzed by indirect immunofluorescence with an anti-FAC polyclonal antibody. Both wild-type and mutant FAC showed a predominantly cytoplasmic pattern in $>95 \%$ of positive cells (Fig. 1). These results demonstrate that the mutation does not lead to any obvious mislocalization of the protein.
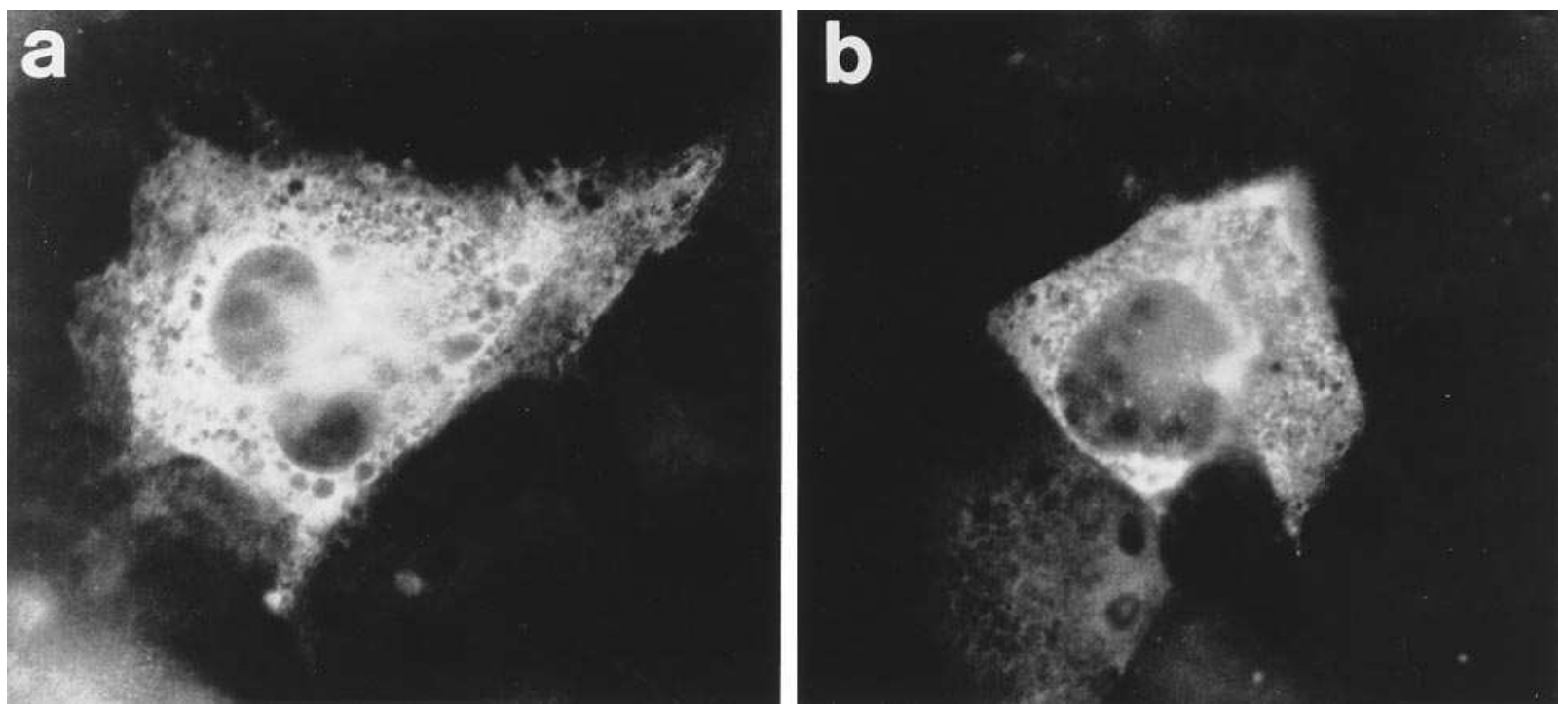

Figure 1. Subcellular location of FAC constructs in transfected COS-1 cells by indirect immunofluorescence. COS-1 cells were transfected with either FAC (a) or FAC-L554P (b), permeabilized with $0.1 \%$ NP-40, and incubated successively with anti-FAC antibody and fluorescein-conjugated goat anti-rabbit IgC. Control incubations of the primary antibody with preimmune serum showed no staining (data not shown). $\times 40$. 
In a previous study, we did not detect any changes in the distribution of wild-type FAC in transiently transfected COS-7 cells after exposure to MMC (6). To evaluate the effect of the L554P mutation on the subcellular distribution of FAC in the presence of crosslinkers, we compared the subcellular distribution of the wild-type and mutant proteins in transfected COS-1 cells by serial immunofluorescence analysis. After exposure of transfected cells to MMC $30 \mathrm{ng} / \mathrm{ml} \mathrm{MMC} \mathrm{for} 15,30,60$, and $120 \mathrm{~min}$, no changes were noted in the subcellular location of either protein (data not shown).

Similar rates of intracellular decay. Another potential mechanism by which the mutation could inactivate the protein is by affecting its intracellular stability. To access for differences in the intracellular half-lives of the proteins, COS-1 cells transfected with either wild-type or mutant FAC were pulselabeled with ${ }^{35} \mathrm{~S}$, and the relative rates of intracellular decay were assessed by chasing the radiolabeled precursors for various times. Cytosolic or nuclear immunocomplexes were analyzed by SDS-PAGE and fluorography, and the $\sim 60-\mathrm{kD}$ band corresponding to FAC was quantitated by PhosphorImaging. A representative experiment is shown (Fig. 2). The intracellular half-life of wild-type FAC was $41 \mathrm{~min}$, while the half-life of the mutant protein was $38 \mathrm{~min}$. In a second experiment, the half-lives of the wild-type and L554P mutant were 46 and 43 min, respectively (data not shown). No translocation into nuclei occurred during the chase period (data not shown). These results demonstrate that the intracellular turnover of FAC is relatively rapid, but the L554P mutation does not appear to affect the intracellular stability of the protein.

Interactions with accessory proteins. We have previously identified several cytoplasmic proteins that bind specifically to a recombinant form of FAC (13). To directly address the effect of the L554P mutation on the ability of FAC to interact with cellular proteins, we generated GST fusion proteins (17) with full-length wild-type FAC or the allele containing the L554P mutation for in vitro binding studies. Both fusion proteins bound to glutathione beads and encoded polypeptides of 85 kD (Fig 3 A). Cell lysates from metabolically labeled 293 cells (14) were then incubated with equivalent amounts of glutathione beads bound to either GST or the fusion proteins. Polypeptides with molecular masses of $69,50,40$, and $30 \mathrm{kD}$ were detected only with the fusion proteins, but not with GST (Fig. 3 B). A number of additional minor bands were also present in the co-precipitates with the fusion proteins, but not with GST alone. All of the interacting polypeptides were detected in cytosolic, but not nuclear, extracts (data not shown), and no obvious differences between the binding pattern of the wild-type and mutant proteins were observed. These results demonstrate that GST fusion proteins can be used to detect a family of cytosolic proteins that bind to FAC. In addition, the L554P mutation does not appear to affect the ability of FAC to bind to these proteins.

Induction of MMC hypersensitivity. The broad biochemical similarities in the behavior of active, wild-type FAC and the inactive L554P mutant suggested a possible strategy of inducing the cellular phenotype of FA in non-FA cells. Overexpression of the L554P mutant in a non-FA cellular background might result in the binding of FABPs to the mutant protein at the expense of the normal endogenous protein. To test this possibility, we generated 293 cells that were stably transfected with either FAC or the mutant construct. Independent G418resistant colonies were isolated and amplified, and their sensitivities to MMC were determined. The expression of the transgenes relative to the endogenous FAC gene was assessed by RT-PCR of cytoplasmic RNA using a common 5' primer and allele-specific $3^{\prime}$ primers. An expected 283-bp fragment was amplified, and the intensity of the bands was estimated by computer scanning of the photograph and densitometry. In every case, the transgene was expressed at four-to-fifteen fold higher levels than the endogenous gene (Fig. 4), as judged by densitometry of the ethidium bromide-stained bands. Relative to the parental or FAC-transfected cells, all four clones ex-
A

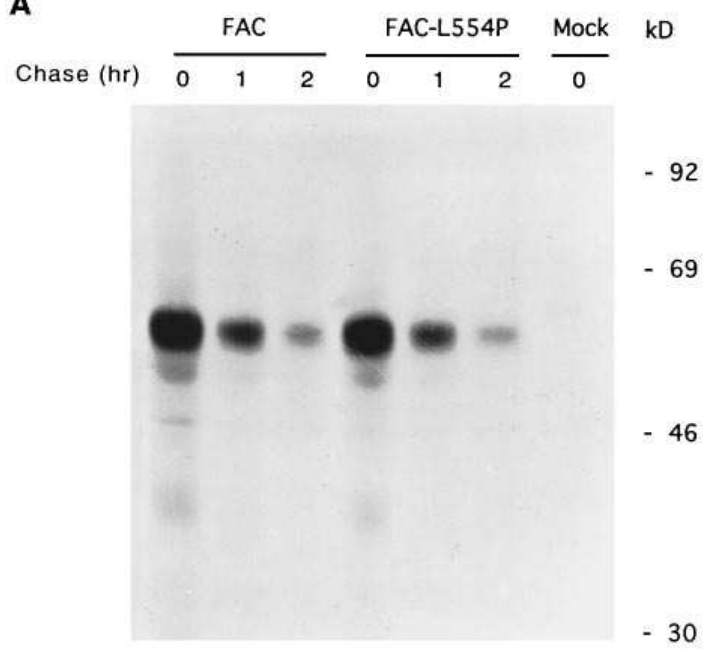

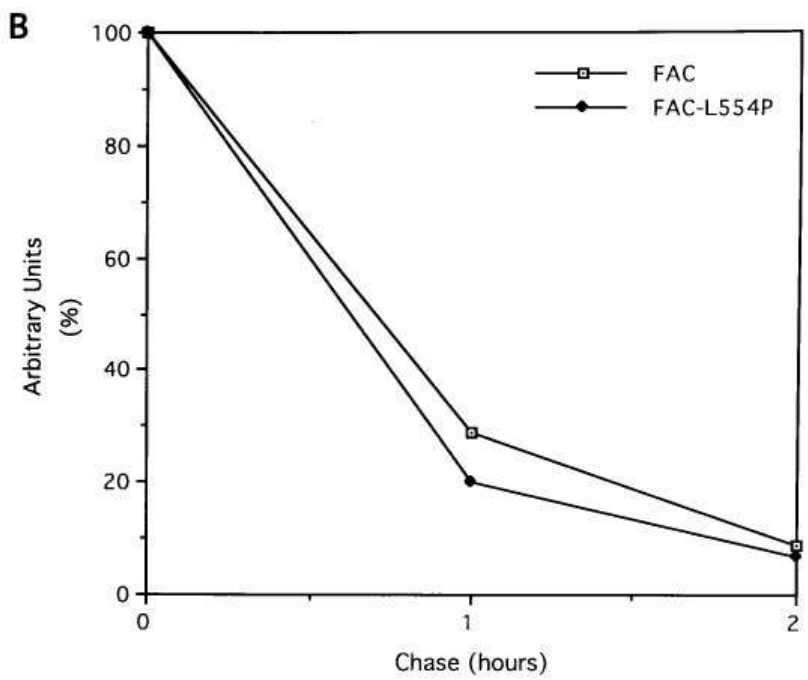

Figure 2. Pulse-chase analysis of FAC polypeptides. (A) COS-1 cells transfected with either FAC or FAC-L554P cloned in pED6 were metabolically labeled for $30 \mathrm{~min}$, washed and analyzed either immediately or chased in radioactive-free medium for 1 and $2 \mathrm{~h}$. Cytosolic extracts were incubated successively with a polyclonal anti-FAC antibody and protein A-agarose beads. Immunocomplexes were boiled in Laemmli buffer in the presence of $100 \mathrm{mM}$ dithiotreitol and analyzed on $10 \%$ SDS-polyacrylamide gels and fluorography. (B) Graphic representation of the autoradiogram in panel A. The intensities of the $60-\mathrm{kD}$ FAC bands were obtained by PhosphorImaging (Molecular Dynamics) and are presented as percentages of the pulse-labeled value. 
A
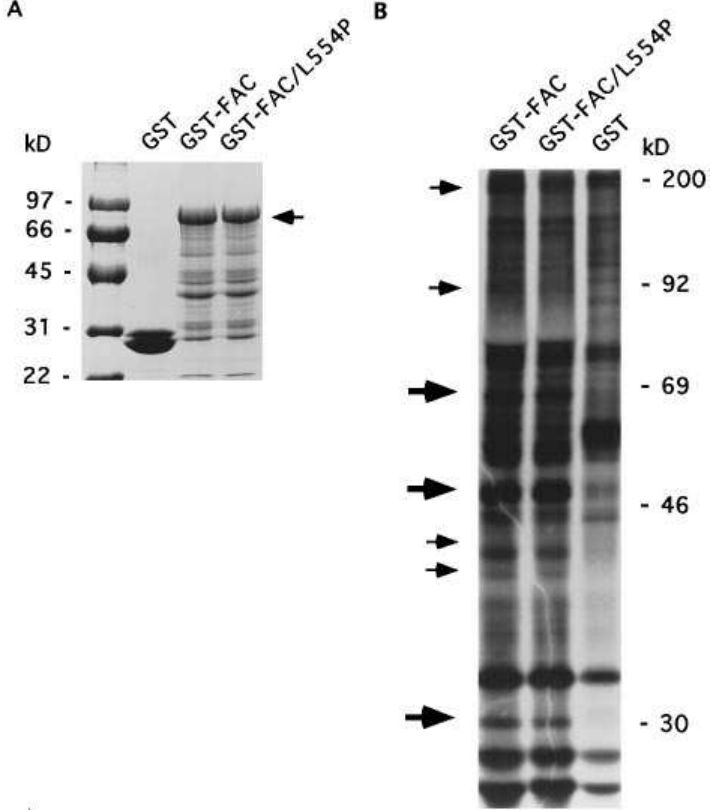

Figure 3. Interaction of FAC with the FABPs in vitro. $(A)$ Aliquots of GST and GST fusions with FAC and FAC-L554P (arrow) were electrophoresed on denaturing $10 \%$ polyacrylamide gel and visualized by Coomassie blue staining. (B) Binding of GST fusion proteins to the FABPs in cytosolic lysates of ${ }^{35}$ S-labeled 293 cells. Bands detected specifically with the GST fusion proteins (small arrows) and those that corresponded in size to the FABPs detected in a previous study with an immunoglobulin fusion protein (large arrows; reference 13) are shown.

pressing the mutant allele were markedly sensitive to the cytotoxic effects of MMC after continuous exposure for $5 \mathrm{~d}$ (Fig. 5). The average doses of MMC reducing survival of sensitive cells to $50 \%$ of control levels (EC50) were $0.9-4.5 \mathrm{ng} / \mathrm{ml}$, while the average EC50 of parental or FAC-overexpressing cells was $34.5 \mathrm{ng} / \mathrm{ml}$. In addition, no differences in the gross morphology

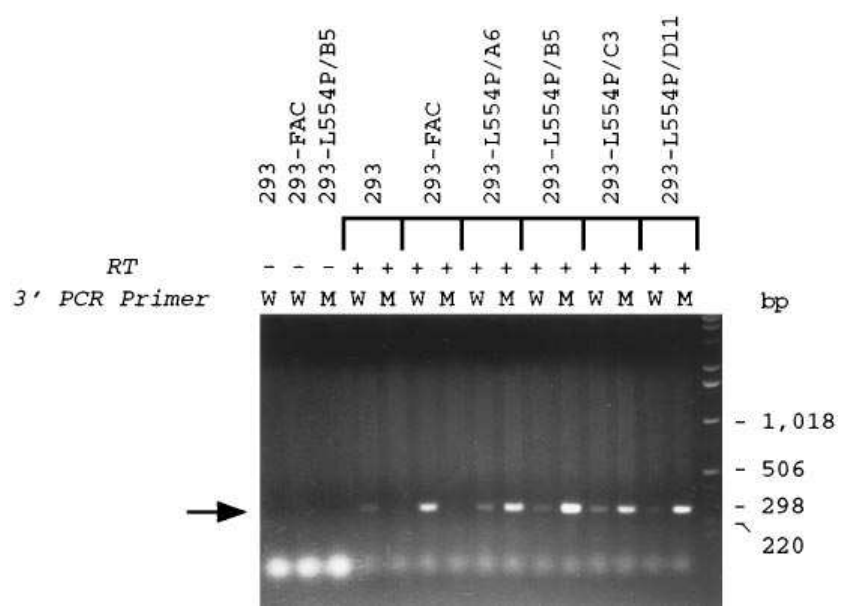

Figure 4. PT-PCR analysis of FAC transcripts in 293 cells. The source of cytoplasmic RNA used for RT-PCR is indicated on top of the figure. In control reactions, reverse transcriptase (RT) was omitted (-). Each PCR reaction was performed using a common 5' primer and an allele-specific $3^{\prime}$ primer corresponding to the wild-type $(W)$ and mutant $(M)$ sequences. The $283 \mathrm{bp}$ amplified fragment is shown (arrow). of 293 cells stably over-expressing these alleles were noted (data not shown). These results demonstrate that overexpression of the L554P mutant in a non-FA genetic background can induce MMC hypersensitivity, the major cellular hallmark of FA.

\section{Discussion}

In this study, we report the biological consequences of the overexpression of a mutant $F A C$ allele in a wild-type cell line, proficient with regard to the FA defect of crosslinker hypersensitivity. Our results lend support to the notion that the function of the FAC polypeptide may be regulated by interactions with other cellular proteins in vivo.

The L554P mutation is the only known natural missense mutation of FAC that can abolish the activity of the protein (7, 12). This mutation has been thought to induce a comformational change in the carboxy-terminal region of FAC, causing the disruption of a predicted $\alpha$-helix as well as a local decrease in hydrophobicity (12). Here we examined experimentally a number of cellular and biochemical parameters that might be altered by this mutation. Using a polyclonal anti-FAC antiserum, Yamashita et al. (5) described a $\sim 60-\mathrm{kD}$ protein in immunoprecipitates of cytosolic extracts from HSC536 cells that was consistent with the predicted size of FAC. These cells belong to FA complementation group $\mathrm{C}$ and are heterozygous for the L554P mutation (7). Because the other mutant allele has not been fully characterized and might also encode an immunoreactive product (Dos Santos, C.C., and M. Buchwald, unpublished observation), the precise origin of the $60-\mathrm{kD}$ polypeptide could not be determined. We expressed the L554P mutation in COS-1 cells and compared the pattern of localization to the wild-type polypeptide. One possible confounding factor in such an analysis may be the immunodetection of the wild-type endogenous polypeptide. However, the expression of FAC is generally quite low in most mammalian cells $(6,7)$, including COS cells (Youssoufian, H., unpublished observation). Thus, with the judicious use of our anti-FAC antibody, the transiently expressing cells are easily distinguished from neighboring negative cells, and the expression of each allele can be evaluated separately with little concern about background staining. Under those conditions, the subcellular location of the mutant or wild-type polypeptides was indistinguishable. In addition, treatment of cells transiently expressing the mutant or wild-type proteins with MMC and serial determinations by indirect immunofluorescence showed no changes in the distribution of these proteins. Thus, the L554P mutation does not appear to cause the mis-localization of the protein.

The potential physico-chemical changes induced by this mutation might also affect the stability of either the transcript or the protein, which could reduce the intracellular levels of FAC by promoting its degradation. Northern analysis of RNA from HSC536 cells compared with a large number of other cell lines shows no differences in the steady state level of the $4.5-\mathrm{kb}$ FAC transcript (Youssoufian H., unpublished observation). Thus, the mutation does not appear to affect the stability of the transcript. We also assessed the effect of the mutation on the stability of the protein by pulse-chase analysis. Although misfolding of the carboxy terminus of the protein could affect the stability of the protein, under our experimental conditions this mutation had no appreciable effect on the intracellular half-life of FAC.

Although there are currently no in vitro functional assays 


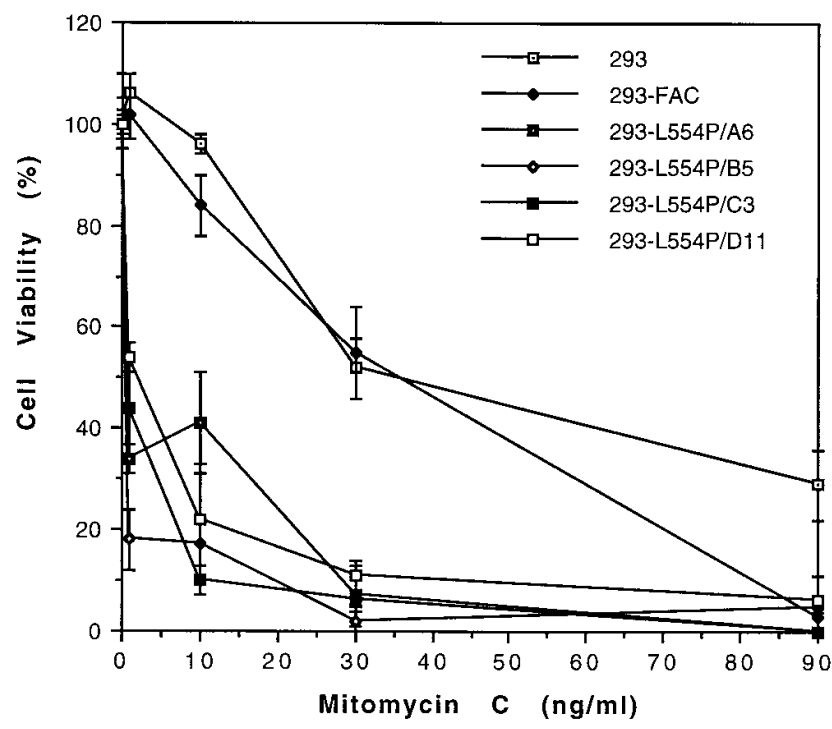

Figure 5. Cellular viability was assessed by trypan blue exclusion after continuous growth in MMC at the indicated doses for $5 \mathrm{~d}$. Each experiment was performed in triplicate.

of FAC, we have recently described the binding of a group of cytosolic proteins to a chimeric immunoglobulin-FAC molecule that was immobilized to protein A-agarose beads (13). To test the effect of this mutation on the interaction of FAC with cellular proteins, we generated GST fusion proteins and compared the ability of the wild-type and mutant FAC proteins to bind to radiolabeled lysates of 293 cells in a modified version of this assay. The sizes of FAC-binding proteins detected by the two versions of this assay are generally comparable. Both assays detect proteins of molecular masses 65-69, 50, and 30$35 \mathrm{kD}$. In addition, the GST fusion proteins also detect a 175$\mathrm{kD}$ protein as well as a number of additional minor bands. Although we cannot exclude the possibility that some of these bands result from associations with impurities in GST fusion proteins, the consistency in the pattern of protein binding with different isolates of GST fusion proteins makes this possibility somewhat less likely. These results lend further support to the protein interaction studies described previously (13). Importantly, no differences in the binding pattern between the wildtype and mutant GST fusion protein were seen, although at least two limitations to this analysis force us to qualify our results. First, this assay is not capable of detecting subtle quantitative differences that may result from variations in binding affinities. Second, proteins that are expressed at low abundance are also likely to escape detection. Thus, it is conceivable that both qualitative and quantitative differences exist between the wild-type and mutant proteins, and one or more such alterations could account for the inactivity of the mutant protein.

The overexpression of the L554 mutation in 293 cells led to an obvious decrease in the survival of these cells after continuous treatment with MMC. The degree of MMC hypersensitivity is similar to that of a number of FA-C cell lines as well as from other groups (7, 13; Youssoufian, H., unpublished). A plausible mechanism for the induction of MMC hypersensitivity is by competition of the mutant protein with the endogenous FAC protein for binding to the FABPs. This conclusion is reinforced by the gross similarity in the profiles of cellular pro- teins that interact with both wild-type and mutant FAC proteins in vitro.

A similar strategy has been used to induce MMC hypersensitivity by overexpression of a mutant DNA repair protein. The human ERCC-1 gene plays an important role in nucleotide excision repair and can complement the repair defect of chinese hamster ovary cells (18). The sensitivity of these cells to ultraviolet light and $\mathrm{MMC}$ is also reminiscent of the human autosomal recessive disease xeroderma pigmentosum (19). Overexpression of a mutant form of the $E R C C-1 \mathrm{cDNA}$ in repair-proficient HeLa cells induced hypersensitivity to both ultraviolet light and MMC (20). Interestingly, the latter effect was more pronounced. It is now clear that ERCC- 1 forms a multimeric complex that is absolutely required for the incision step of nucleotide excision repair (21-24). The induction of the mutant phenotype is consistent with the competition model proposed for FAC. This approach should be suitable for the in vivo mapping of cellular proteins that bind to particular domains of the FAC polypeptide in both established cell lines and primary hematopoietic cells. Overexpression of mutant $F A C$ alleles might also lead to the generation of animal models of FA by transgenic approaches.

Mutations that involve the extreme carboxy-termini of proteins infrequently give rise to phenotypic changes (25). It is interesting that a number of such mutations cause a more severe phenotype than similar mutations in other regions of the protein (26). The L554P mutation is one of two different mutations that affect the carboxy-terminal portion of FAC. Another point mutation in exon 14 of FAC changes the arginine residue at codon 548 into a stop codon (8), which results in the predicted truncation of eleven amino acids from the carboxyterminus. The effect of this mutation on the severity of the disease is not yet known. However, it appears that the L554P mutation is associated with a relatively severe phenotype (10). It is tempting to speculate that the severity of this mutation compared with more proximal truncations of the protein is due to its ability to bind FABPs and make them unavailable for other possible cellular activities.

\section{Acknowledgments}

We thank Dr. Robert Wise for providing us the pED6 vector.

This study was supported by grants from the National Institutes of Health (HL-52138), the March of Dimes Birth Defects Foundation (6-FY95-0279), the Aplastic Anemia Foundation of America, the Medical Research Council of Canada and the Genetic Diseases Network of Centre of Excellence.

\section{References}

1. Liu, J.M., M. Buchwald, C.E. Walsh, and N.S. Young. 1994. Fanconi anemia and novel strategies for therapy. Blood. 84:3995-4007.

2. Setlow, R.B. 1978. Repair deficient human disorders and cancer. Nature (Lond.). 271:713-717.

3. Strathdee, C.A., A.M.V. Duncan, and M. Buchwald. 1992. Evidence for at least four Fanconi anaemia genes including FACC on chromosome 9. Nature Genet. 1:196-198.

4. Joenje, H., J.R. Lo Ten Foe, A.B. Oostra, C.G.M. van Berkel, M.A. Rooimans, T. Schroeder-Kurth, R.-D. Wegner, J.J.P. Gille, M. Buchwald, and F. Artwert. 1995. Classification of Fanconi anemia patients by complementation analysis: evidence for a fifth genetic subtype. Blood. 86:2156-2160.

5. Yamashita, T., D.L. Barber, Y. Zhu, N. Wu, and A.D. D'Andrea. 1994. The Fanconi anemia polypeptide FACC is localized to the cytoplasm. Proc. Natl. Acad. Sci. USA. 91:6712-6716.

6. Youssoufian, H. 1994. Localization of Fanconi anemia C protein to the cytoplasm of mammalian cells. Proc. Natl. Acad. Sci. USA. 91:7975-7979. 
7. Strathdee, C.A., H. Gavish, W.R. Shannon, and M. Buchwald. 1992. Cloning of cDNAs for Fanconi's anaemia by functional complementation. $\mathrm{Na}$ ture (Lond.). 356:763-767.

8. Murer-Orlando, M., J.C. Lierena, Jr., F. Birjandi, R.A. Gibson, and C.G. Mathew. 1993. FACC gene mutations and early prenatal diagnosis of Fanconi's anaemia. Lancet. 342:686.

9. Whitney, M.A., H. Saito, P.M. Jakobs, R.A. Gibson, R.E. Moses, and M. Grompe. 1993. A common mutation in the FACC gene causes Fanconi anaemia in Ashkenazi Jews. Nature Genet. 4:202-205.

10. Verlander, P.C., J.D. Lin, M.U. Udono, Q. Zhang, R.A. Gibson, C.G. Mathew, and A.D. Auerbach. 1994. Mutation analysis of the Fanconi anemia gene FACC. Am. J. Hum. Genet. 54:595-601.

11. Gibson, R.A., A. Hajianpour, M. Murer-Orlando, M. Buchwald, and C.G. Mathew. 1993. A nonsense mutation and exon skipping in the Fanconi anaemia group C gene. Hum. Molec. Genet. 2:797-799.

12. Gavish, H., C.C. dos Santos, and M. Buchwald. 1993. A Leu554-to-Pro substitution completely abolishes the functional complementing activity of the Fanconi anemia (FACC) protein. Hum. Mol. Genet. 2:123-126.

13. Youssoufian, H., A. D. Auerbach, P.C. Verlander, V. Steimle, and B. Mach. 1995. Identification of cytosolic proteins that bind to the Fanconi anemia complementation group $\mathrm{C}$ polypeptide in vitro: Evidence for a multimeric complex. J. Biol. Chem. 270:9876-9882.

14. Graham, F.L., J. Smiley, W.C. Russell, and R. Nairn. 1977. Characteristics of a human cell line transformed by DNA from human adenovirus type 5.J. Gen. Virol. 36:59-72.

15. Davies, M.V., and R.J. Kaufman. 1992. The sequence context of the initiation codon in the encephalomyocarditis virus leader modulates efficiency of internal translation initiation. J. Virol. 66:1924-1932.

16. Harlow, E., and D. Lane. 1988. Antibodies. Cold Spring Harbor Laboratory Press, Cold Spring Harbor, New York

17. Frangioni, J.V., and B.G. Neel. 1993. Solubilization and purification of enzymatically active glutathione $S$-transferase (pGEX) fusion proteins. Anal. Biochem. 210:179-187.

18. Westerveld, A., J.H.J. Hoeijmakers, M. van Duin, J. de Wit, H. Odijk, A. Pastink, R.D. Wood, and D. Bootsma. 1984. Molecular cloning of a human DNA repair gene. Nature (Lond.). 310:425-429.

19. Arlett, C.F. and Lehmann, A.R. 1978. Human disorders showing increased sensitivity to the induction of genetic damage. Annu. Rev. Genet. 12:95115 .

20. Belt, P.B.G.M., M.F. van Oosterwijk, H. Odijk, J.H.J. Hoeijmakers, and C. Backendorf. 1991. Induction of a mutant phenotype in human repair proficient cells after overexpression of a mutated human DNA repair gene. Nucleic Acids Res. 19:5633-5637.

21. Van Vuuren, A.J., E. Appeldoorn, H. Odijk, A. Yasui, N.G. Jaspers, D. Bootsma, and J.H. Hoeijmakers. 1993. Evidence for a repair enzyme complex involving ERCC1 and complementing activities of ERCC4, ERCC11 and xeroderma pigmentosum group F. EMBO (Eur. Mol. Biol. Org.) J. 12:3693-3701.

22. Li, L., S.J. Elledge, C.A. Peterson, E.S. Bales, and R.J. Legerski. 1994 Specific association between the human DNA repair proteins XPA and ERCC1. Proc. Natl. Acad. Sci. USA. 91:5012-5016.

23. Li, L., C.A. Peterson, X. Lu, and R.J. Legerski. 1995. Mutations in XPA that prevent association with ERCC1 are defective in nucleotide excision repair. Mol. Cell. Biol. 15:1993-1998.

24. Van Vuuren, A.J., E. Appeldoorn, H. Odijk, S. Humbert, V. Moncollin, A.P. Eker, N.G. Jaspers, J.M. Egly, and J.H. Hoeijmakers. 1995. Partial characterization of the DNA repair protein complex, containing the ERCC1, ERCC4, ERCC11 and XPF correcting activities. Mutat. Res. 337:25-39.

25. Cooper, D.N., and M. Krawczak. 1993. Human gene mutation. Bios Scientific, Oxford, UK.

26. Gal, A., A. Artlich, M. Ludwig, G. Niemeyer, K. Olek, E. Schwinger, and A. Schinzel. 1991. Pro-347-Arg mutation of the rhodopsin gene in autosomal dominant retinitis pigmentosa. Genomics. 11:468-470. 